\title{
Impact of Acculturation on Body Mass Index in Haitians
}

\author{
Nirva Berthold Lafontant, $\mathrm{PhD}, \mathrm{RN}$ \\ Walden University, Minnesota, United States
}

Contact: nirvaberthold@comcast.net

\begin{abstract}
Longer term immigrants residing in the United States exhibit physical health decline related to higher body mass index (BMI). Theories on immigrant acculturation have been used to examine health patterns by length of stay in the United States. The purpose of this cross-sectional study, guided by the Schwartz model of acculturation, was to examine the effect of acculturation and length of stay in the United States on BMI in a sample of Haitian immigrants living in a northeast metropolitan area. The research question was developed to examine the effects of acculturation and how long immigrants reside in the United States on BMI. The Participants included a convenience sample of 116 Haitian men and women, aged 18 years and older, who had relocated to the United States for 3 years or more. Data were collected using a demographic questionnaire and medical records from a participating health clinic and then analyzed by conducting multiple linear regression statistical analyses. Results revealed that acculturation, length of stay in the United States, age, gender, and physical activity were not significant predictors of BMI change. An ancillary analysis using the subscales of acculturation revealed similar results. This study may provide positive social change by enabling health providers to understand the beliefs, values, and practices of Haitian immigrant groups and the acculturation pattern of individuals when providing care for this population.
\end{abstract}

Keywords: acculturation, bidimensional, body mass index, BMI change, Haitian immigrant population, length of stay in the United States

Date Submitted: June 22, 2019 | Date Published: September 27, 2019

Recommended Citation

Berthold Lafontant, N. (2019). Impact of acculturation on body mass index in Haitians. Journal of Excellence in Nursing and Healthcare Practice, 1, 23-35. https://doi.org/10.5590/JENHP.2019.1.1.04

\section{Introduction}

Obesity is a health issue in the United States, affecting people of all races and ethnic groups. The Centers for Disease Control and Prevention (CDC) began collecting data on body mass index (BMI) in the mid-1980s. Data showed that in 2015-2016, the prevalence of obesity in the United States was 39.8\% (CDC, 2018). Obesity, as defined by the CDC (2016), is any weight that is considered unhealthy at a given height. In measuring obesity, the standard measure has been through the use of the BMI, which uses a calculation of

Note: I am sincerely grateful and would like to thank Dr. Janice Long, Dr. Leslie Hussey, and Dr. Diane Whitehead for their guidance and support throughout my dissertation. Dr. Long, I have learned a great deal from your mentorship and hope to make you proud through my mentoring of others one day. I also thank the physician and administrative staff at the health clinic for making it possible for me to conduct and complete my study. I will forever be grateful for your dedication to see me through the end of my dissertation. 
body fat percentage, dividing the square of a person's height and weight (CDC, 2016). A person is obese if the $\mathrm{BMI}$ is $\geq 30$ (CDC, 2013). Other measures of obesity have been recommended. For example, waist circumference is another screening tool that is used to measure body weight status. According to the CDC (2013), excess body fat is indicated if the waist circumference is $>101.6 \mathrm{~cm}$ for a man or $>88.9 \mathrm{~cm}$ for nonpregnant women.

Although measurement strategies are not taken to diagnose obesity-related conditions, the nature of obesity has been established. Obesity places millions at risk for heart disease, stroke, diabetes, and other complications. Complications of being overweight or obese take a toll on the health of all populations (Cruz, Hernandez-Lane, Cohello, \& Bautista, 2013). However, the prevalence of obesity and its complications are greater among minority groups than nonminority groups who are obese, and the 2016 census report identified 29.2\% of the population as minorities (Lind et al., 2016). Although U.S. immigrants enter the country with a lower incidence of being overweight, the prevalence of obesity rose with increased length of habitation in the United States (Afable-Munsuz, Mayed, Perez-Stable, \& Haan, 2014).

One minority group that has been impacted by obesity is the Haitian community in the United States. Schulz and Batalova (2017) reported the Haitian population at 200,000 in 1990 and 606,000 in 2012. As a result of the 2010 earthquake, Haitians are one the fastest growing Caribbean immigrant populations in the United States (Lubetkin et al., 2015). The Haitian Caribbean population now represents $1.5 \%$ of the overall foreignborn population in the United States (Schulz \& Batalova, 2017).

Generally, both weight and cardiovascular disease tend to rise with longer length of stay in the United States for all immigrants (Lê-Scherban et al., 2014). Afable-Munsuz et al. (2014) found that although some immigrants enter the country with a low frequency of being overweight, the occurrence of obesity and diabetes rises with increased length of habitation in the United States. Because a high BMI is prevalent in the United States, Haitian immigrants may experience high BMI over time as they acculturate to American lifestyles. Increased BMI may also be linked to changes in socioeconomic status for Haitian immigrants because they make more money in the United States than in Haiti, potentially leading to increased food consumption.

\section{Purpose}

The purpose of this quantitative, cross-sectional study was to examine the effect of acculturation and length of stay in the United States on BMI in a sample of Haitian immigrants living in a large metropolitan city in the Northeast. Acculturation is defined as the process of experiencing changes in a cultural pattern with continuous and prolonged exposure to a new culture (Agbemenu, 2016; Archuleta, 2015; McDermott-Levy, 2009). Having a good understanding of acculturation can help in the promotion of health-related initiatives and culturally competent care to immigrant communities.

Research Question: What effect does acculturation and length of stay in the United States have on BMI among Haitians who have immigrated to a large metropolitan city in the Northeast?

$H_{o}$ : Acculturation and length of stay in the United States have no effect on BMI among Haitians who have immigrated to a large metropolitan city in the Northeast.

$H_{a}$ : Acculturation and length of stay in the United States have an effect on BMI among Haitians who have immigrated to a large metropolitan city in the Northeast. 


\section{Significance}

Vulnerable populations face early death due to chronic diseases, low socioeconomic status, and lack of resources and education (Flaskerud \& Winslow 2010; Nyamathi, Koniak-Griffin, \& Greengold, 2007). Lu, Donglan, van Meijgaard, MacLeod, and Fielding (2015) identified that immigrant health outcomes can be influenced based on their level of acculturation. Life-threatening financial instability, sociopolitical events, and hopelessness have driven many Haitians to relocate to other countries, predominantly the United States (Allen, Marcelin, Schmitz, Hausmann, \& Shultz, 2012). Scholars have not examined the effect of acculturation on weight and BMI in this vulnerable population; therefore, there was a need for research on acculturation and BMI among Haitian immigrants in a large metropolitan city in the northeast United States. This study contributed to knowledge of how acculturation is associated with health issues The study may initiate an advocacy role from health care providers to promote policy changes that will enhance screening programs to become more culturally competent with the use of acculturation instruments, such as the Stephenson Multigroup Acculturation Scale (SMAS; Stephenson, 2000), at the bedside or in the communities.

The findings of this research will lead to long-term positive social change by facilitating an increase in knowledge for nurses and for nursing as a discipline. The results may raise awareness of the importance of measuring acculturation and its association with health behaviors. In addition, the study results may contribute to the understanding of providing transcultural, competent, and quality care to the Haitian immigrant population.

\section{Theoretical Framework}

The Schwartz model of acculturation guided this study. The model includes immigrants' cultural identity within cultural groups and their attachments, such as cultural values and cultural practices (Ro \& Bostean, 2015). The SMAS examined the four mediators that come from the Schwartz model of acculturation: (a) ethnic and culture identity, (b) cultural values (family cohesion), (c) cultural practices (English language proficiency), and (d) acculturation stress. Acculturation has been shown to have a relationship with factors related to sociocultural adaptation, cultural identity, family cohesion, and acculturation stress (Gaskins, Baskin, \& Person, 2012; Kuo, 2014; Weisskirch, 2013).

\section{Literature Review}

According to Cruz et al. (2013), obesity is a health issue in the United States; its long-term effects can cause extra stress on the heart, lung functions, and joint functions, and it may also alter the functions of many other internal mechanisms. Obesity prevalence is greater among minority groups than nonminority groups in the United States (Hofmann, 2016). Scholars have examined the impact of acculturation and length of residency of immigrants in the United States (Lu et al., 2015). Although immigrants enter the country with a low frequency of being overweight, the occurrence of obesity and diabetes rises with increased length of habitation in the United States (Afable-Munsuz et al., 2014). Tsujimoto, Kajio, and Sugiyama (2016) assessed the associations between the length of time in the United States and the prevalence of obesity and diabetes. The sample included adults $(n=37,639)$ and children/adolescents $(n=28,282)$. Tsujimoto et al. found that obesity and diabetes increased with length of time in the United States in the adults and for children/adolescents; greater than 20 years in the United States showed a significant association with higher obesity rates. The study did not address the Haitian population.

Xi, Takyi, and Lamptey (2015) found a significant increase in initial BMI in the Asian and Hispanic immigrant population as the length of time in the country increased; yet the researchers did not address the Haitian 
population. Nguyen, Smith, Reynolds, and Freshman (2015) examined the relationship between obesity and acculturation in foreign-born Asians in the United States and found that the hypothesized links of measuring acculturation with both variables positively predicted obesity in the Filipino subgroup. These studies were conducted on Latino, Mexican, African, Asian, and Korean immigrant populations concerning the effect of acculturation and length of stay in the United States on BMI. However, studies have not been done on the relationship between weight and BMI and longer U.S. stay in the Haitian immigrant population.

\section{Method}

This study was a quantitative, cross-sectional design. The target group number of this study was 116 participants ( $n=54$ men and 62 women), aged 18 to 78 . Data were collected using a sociodemographic questionnaire and the SMAS. A convenience sample of Haitian men and women who had been in the country for 3 years or more was included. The network that was used to recruit the participants was the health clinic of a local physician and medical associates, located in a large metropolitan city in the Northeast. Those eligible to participate in the study included men and women aged 18 and up who were born in Haiti and who had resided in the northeast metropolitan area for at least 3 years. Participants received a written consent form, as well as a demographic survey and the SMAS questionnaire. They needed to be able to read and understand the materials in English. Approval was granted from Walden University Institutional Review Board.

Length of stay in the United States and acculturation were examined in the context of weight or BMI. In addition to acculturation and length of time in the United States, age, gender, and physical activity were the covariates. A demographic questionnaire containing five items to determine age, gender, height, years in the United States, and activity levels was administered after the consent was obtained. The data from the participants' medical records were obtained from the clinic to examine BMI at the initial visit and when the questionnaire was administered.

The SMAS (Stephenson, 2000) was used to measure acculturation, which was the independent variable. The SMAS consists of 32 items divided into two subscales: Ethnic Society Identification (ESI), which consists of 18 items, and Dominant Society Identification (DSI; Stephenson, 2000), which has 14 items. The items in each subscale measure the domains of attitude/language, behavior/food, media, and interaction. Participants rated each item using a Likert scale ( $1=$ false, $2=$ partly false, $3=$ partly true, and $4=$ true); this resulted in a total acculturation score and two subscale scores. To arrive at the mean item response, the scores for each item were summed and then divided by the number of questions (i.e., 32). A low score indicated greater acculturation to host culture or very little cultural identity, whereas a high score indicated a well-established orientation to ethnic society or high cultural identity (Stephenson, 2000). Stephenson demonstrated the validity of the SMAS across multiple studies using exploratory factor analysis and correlations with related measures. The exploratory factor analysis supported a two-factor solution with all item loadings exceeding 0.5. Additionally, the SMAS subscales were significantly correlated with the Acculturation Rating Scale for Mexican Americans-II and the Bidimensional Acculturation Scale for Hispanics in a Hispanic population. The SMAS has a coefficient alpha of .86, demonstrating strong reliability (Stephenson, 2000).

\section{Data Analysis}

SPSS Version 25 was the software used in this analysis. To avoid missing data prior to running the analysis, a table that contains the variable names and values was created in Microsoft Excel. $Z$ scores and standardized scores were created under the descriptive tabs in SPSS to search for outliers, which are scores that exceed the value of the variables and that can cause both Type 1 and Type 2 errors. The six main predictors were age, gender, height, physical activity, acculturation, and the length of stay in the United States. For these 
predictors, the dependent variable was BMI. To evaluate the correlation between BMI and age, gender, height, physical activity, acculturation, and the length of stay in the United States, linear multiple regression analysis was used (Field, 2013). The a priori sample size calculator $\mathrm{G}^{*}$ Power Version 3.1.9 (Faul, Erdfelder, Buchner, \& Lang, 2014) was used in multiple linear regression to measure the strength of the correlations between the six predictor variables on the dependent variable in a sample of 116 participants.

\section{Results}

The effect sizes of independent variables on the dependent variable were assessed, and the recruitment of participants included Haitian men and women in an effort to reflect the targeted population. Because a priori power analyses used for sample size estimations only estimate effect size, the actual power achieved can be different than what was estimated (Ialongo, 2016). Every individual sample will produce a unique effect size (Ialongo, 2016). Post hoc power analyses indicated that the achieved power of the regression with 116 participants was 0.51 , and the achieved power of the regression with outliers removed from the analysis $(n=3$ outliers removed for a total of 113 participants used in the analysis) was similar at 0.50, as suggested by Faul et al. (2014). There was a reduced ability to detect a statistical relationship, if one existed. Because the post hoc power analysis indicated low power (0.51), a Type 2 error may have occurred, resulting in a failure to reject the null hypothesis (i.e., that acculturation and length of stay in the United States have no effect on BMI) when a relationship existed.

Of the 116 participants, $53 \%$ were female $(n=62)$. Participants responded to a question asking about their level of activity. More than half of the participants indicated that they were active sometimes $(n=52,44.8 \%)$ or very often ( $n=22,18.9 \%$ ), making up the largest percent of responses on the question. A majority of participants had lived in the United States 10 years or more $(n=100,86 \%)$. Full frequencies and percentages are presented in Table 1.

Table 1. Frequencies and Percentages of Gender, Physical Activity, and Length in the United States $(\mathrm{n}=116)$

\begin{tabular}{lrr}
\hline Variable & $\boldsymbol{n}$ & $\mathbf{\%}$ \\
\hline Gender & 62 & \\
Female & 54 & 46.45 \\
Male & 0 & 0.00 \\
Missing & & \\
Physical activity & 42 & 36.21 \\
$\quad$ Not at all & 52 & 44.83 \\
Sometimes & 22 & 18.97 \\
Very often & 0 & 0.00 \\
Missing & & \\
Length of time in the United States & 11 & 9.48 \\
3-5 Years & 5 & 4.31 \\
5-10 Years & 100 & 86.21 \\
10 Years or more & 0 & 0.00 \\
Missing & & \\
\hline
\end{tabular}

Note. Due to rounding errors, percentages may not equal 100\%.

Baseline measures were taken from the 2015 health records (T1). The participants were, on average, 51.76 years old $(S D=14.42$ years $)$ and their average height was $166.16 \mathrm{~cm}(S D=9.82 \mathrm{~cm})$. Participants' average T1 
weight was $73.27 \mathrm{~kg}(S D=17.01 \mathrm{~kg})$, and their average weight when the questionnaire was administered (T2) was $83.76 \mathrm{~kg}(S D=17.33 \mathrm{~kg})$. Participants' average T1 BMI was $26.83 \mathrm{~kg} / \mathrm{m}^{2}\left(S D=6.79 \mathrm{~kg} / \mathrm{m}^{2}\right)$, and their average T2 BMI was $30.60 \mathrm{~kg} / \mathrm{m}^{2}\left(S D=6.67 \mathrm{~kg} / \mathrm{m}^{2}\right)$. These results are summarized in Table 2 .

Table 2. Means, Medians, and Standard Deviations of Age, Weight, Height, and Body Mass Index $(B M I)$

\begin{tabular}{|c|c|c|c|c|c|c|c|}
\hline \multirow[b]{2}{*}{ Measure } & \multirow[b]{2}{*}{ Age } & \multicolumn{2}{|c|}{ Weight, kg } & \multirow{2}{*}{$\begin{array}{c}\text { Height, } \\
\text { cm }\end{array}$} & \multicolumn{3}{|c|}{ BMI, $\mathbf{k g} / \mathbf{m}^{2}$} \\
\hline & & T1 & T2 & & T1 & T2 & Change \\
\hline$M$ & 51.76 & 73.27 & 83.76 & 166.16 & 26.83 & 30.60 & -3.69 \\
\hline$M d n$ & 51.00 & 72.12 & 81.65 & 165.10 & 25.65 & 29.77 & -1.86 \\
\hline$S D$ & 14.42 & 17.01 & 17.33 & 9.82 & 6.79 & 6.67 & 5.01 \\
\hline
\end{tabular}

Note. $n=114$ for BMI change, all other $n=116$. T1 $=$ Time 1, baseline in 2015; T2 = Time 2, when questionnaire was administered.

The 32-item SMAS was ranked on a 4-point Likert scale: Scores ranging from 1.00 to 2.50 indicated high acculturation, scores of 2.50 to 3.00 indicated lower acculturation, and scores above 3.00 indicated low very acculturation (Stephenson, 2000). The average for the total acculturation score was $2.98(S D=0.25)$, suggesting that participants had somewhat low total acculturation. The average of the DSI subscale was 2.32 $(S D=0.59)$, indicating that participants had somewhat higher DSI acculturation than total or ESI acculturation. The average of the ESI subscale was $3.50(S D=0.18)$, indicating very low ESI acculturation. The full range, means, and standard deviations are summarized in Table 3. The ESI subscale had a Cronbach's $\alpha=.58$. The second subscale, DSI, had a Cronbach's $\alpha=.86$. The total acculturation score had a Cronbach's $\alpha=.71$.

Table 3. Means and Standard Deviations of Stephenson Multigroup Acculturation Scale Scores

\begin{tabular}{llll}
\hline Subscale & $\boldsymbol{n}$ & $\boldsymbol{M}$ & $\boldsymbol{S D}$ \\
\hline Ethnic Society Identity & 115 & 3.50 & 0.18 \\
Dominant Society Identity & 116 & 2.32 & 0.59 \\
Total acculturation score & $\mathbf{1 1 6}$ & 2.98 & $\mathbf{0 . 2 5}$ \\
\hline
\end{tabular}

Note. Ethnic Society Identity $n=115$ after removing one outlier.

To address the research question, a multiple linear regression statistical analysis was conducted to explain the relationship between the predictor variables, which were acculturation, length of stay in the United States, and BMI change. Prior to conducting the regression, the assumptions of normality, homoscedasticity, and absence of multicollinearity were assessed. Normality was evaluated using a $Q-Q$ scatterplot, as suggested by Bates, Mächler, Bolker, and Walker (2014). Normality can be assumed if the data points generally conform to the diagonal normality line. The scatterplot shows deviation from normality. However, minor deviations from normality can be accepted, as the $F$ test used in regressions is robust against violations of the normality assumption provided the sample size is large ( $n>50$; Pituch \& Stevens, 2015).

For this analysis, the continuous predictor variable was acculturation score. The covariates were age, gender, physical activity, and length of stay. As physical activity and length of stay were categorical variables with more than two categories, the variables were dummy-coded for entry into the regression model. Physical activity was dummy-coded into the variables sometimes and very often, with not at all as the reference category. Length of stay was dummy-coded into the variables $5-10$ years and 10 years or more, with $3-5$ years as the reference category. The continuous dependent variable was BMI change. BMI change was 
calculated by subtracting the participants' T2 weight with their T1 weight to show if there was a positive (i.e., increase) or a negative (i.e., decrease) change in BMI.

The results of this linear regression model were not significant, $F(8,104)=0.96, p=.475, R^{2}=0.07$. The combination of age, gender, physical activity, length of stay, and the subscales of acculturation (DSI and ESI) were not significantly related to BMI change. Additionally, no individual predictor was significant, and the null hypothesis could not be rejected through this ancillary analysis. Table 4 summarizes the results of this regression model.

Table 4. Results for Linear Regression With Age, Gender, Activity, Length, and Acculturation Score Predicting Body Mass Index Change

\begin{tabular}{lccccc}
\hline Variable & $\boldsymbol{B}$ & $\boldsymbol{S E}$ & $\boldsymbol{\beta}$ & $\boldsymbol{p}$ & $\mathbf{9 5 \%} \mathbf{C I}$ \\
\hline (Intercept) & 6.93 & 7.29 & 0.00 & .344 & {$[-7.52,21.37]$} \\
Age & 0.04 & 0.04 & 0.13 & .286 & {$[-0.04,0.13]$} \\
Gender (ref: female) & 0.62 & 1.02 & 0.06 & .543 & {$[-1.40,2.65]$} \\
$\quad$ Male & & & & & \\
Physical activity (ref: not at all) & & & & & \\
$\quad$ Sometimes & -0.11 & 1.09 & -0.01 & .918 & {$[-2.26,2.04]$} \\
$\quad$ Very often & 1.11 & 1.39 & 0.09 & .427 & {$[-1.65,3.87]$} \\
Length of stay (ref: 3-5 years) & & & & & \\
$\quad$ 5-10 Years & -0.14 & 2.96 & -0.01 & .962 & {$[-6.02,5.74]$} \\
$\quad$ 10 Years or more & -2.04 & 1.84 & -0.14 & .269 & {$[-5.69,1.60]$} \\
Acculturation score & -3.90 & 2.20 & -0.20 & .080 & {$[-8.27,0.47]$} \\
\hline
\end{tabular}

Note. CI $=$ confidence interval; ref $=$ reference.

\section{Discussion}

This study was designed to examine the relationship between acculturation and length of stay in the United States and how they affect BMI in a sample of 116 Haitian immigrants living in a large northeastern metropolitan area. Four mediators from the Schwartz model were used: cultural practices (English language proficiency), ethnic and cultural identity, cultural values (family cohesion), and acculturation stress. Scholars have revealed associations between length of stay in the United States, acculturation, and BMI changes (Afable-Munsuz et al., 2014; Lê-Scherban et al., 2014; Oza-Frank, Stephenson, \& Venkat Narayan, 2011; Ro \& Bostean, 2015; Venkatesh, Weatherspoon, Kaplowitz, \& Song, 2013). Mediators such as English language proficiency and acculturation stress were found in the literature as contributors to weight gain. However, in the current study, English language was a component of the DSI subscale, while acculturation stress was a component of the ESI subscale. Results revealed there was no relationship between BMI and acculturation. Haitian immigrants surveyed scored higher on the EIS compared to the DSI scale. Haitians in this study had retained more of their ethnic identity rather than acculturate to the dominate society identity, although no statistically significant differences were found between the relationship of acculturation to BMI. This could be due to the various factors that contribute to acculturation that immigrants experience when arriving and eventually settling into a new country. In terms of acculturation at a group level, immigrants gradually adapt to cultural practices, institution, and social structures, where adaptation is reached to lessen conflicts between patterns of host culture and country of origin (Agbemenu, 2016; Buscemi, 2011). At the individual level, 
acculturation is achieved in personal behaviors and values with continued contact with the host culture (Zhang \& Tsai, 2014).

In this dual-role process, immigrants choose one of four ways to acculturate into the host nation: assimilation (when immigrants embrace the host nation's culture wholeheartedly), separation (where immigrants separate themselves from the dominant culture to preserve their original culture), integration (where immigrants participate in the social system yet honor their original culture), and marginalization (where immigrants lose interest and become alienated from both cultures; Agbemenu, 2016; Buscemi, 2011).

The findings of this study provided support for the Schwartz model that views acculturation as a process of transforming cultural identity. According to Schwartz et al. (2013), acculturation of first-generation immigrants is the process of adapting to new values, culture, and practices. First-generation immigrants are challenged with discerning which practices, values, and behaviors to keep or reject from the new culture (Schwartz et al., 2013). This was prevalent in the findings, as participants all retained cultural ties to the homeland, thereby rejecting certain behaviors and norms of the host country.

\section{Limitations}

Convenience sampling was used as the sampling strategy because it gave more opportunity to access the sample size needed for the study. Cross-sectional studies are limited because they are conducted at various points in time, which does not provide sufficient evidence as to when participants were exposed to the change that might have caused a negative outcome (Quick \& Hall, 2015). Therefore, it is not possible to determine causation. As this was a nonprobability strategy, the findings of this study cannot be generalized as an evaluation that represents the population. Generalizability may also be limited as changes in BMI only reflected participants who had been in the country for at least 3 years. After a post hoc power analysis, it was found that the statistical power of the study was a limitation. Based on an a priori sample size calculation, it was originally assumed that there would be a power of .80 , based on an estimated medium effect size used within the calculation. However, a medium effect size was not found, and the achieved power of the analysis for this sample was low, at .51. There was a reduced ability to detect a statistical relationship, if one existed. Because the post hoc power analysis indicated low power (0.51), a Type 2 error may have occurred resulting in a failure to reject the null hypothesis when a difference existed.

\section{Recommendations}

Based on the findings of this study, there are multiple recommendations for future research. The first recommendation is that future researchers collect data from the Haitian immigrant population living in areas outside of a metropolitan area of the United States, rather than focus solely on the metropolitan areas. In doing so, researchers can increase the sample size for generalization of the study results. The second recommendation is for my study to be replicated to include a larger sample size; different length of time in the United States; and for the correlation between acculturation, length of time in the United States, and BMI to be measured prospectively and retrospectively.

Dietary habits, level of education, and economic status should also be examined as covariates in future studies on this topic. Although the SMAS used in the study already separated dominant ethnic group identity into subscales, it is recommended that researchers use their own questions that reflect family cohesion and English language to understand acculturation better as this survey was not designed to use the items in the subscales as individual items. The final recommendation is to consider future studies to generalize beyond the local population of Haitians because there are not enough studies on the Haitian community. It may be possible to use a larger sample that may be representative of Haitians. 


\section{Implications for Positive Social Change}

The findings of this study can be used to make health practitioners aware of acculturation and the effect it can have on an individual's adjustment to the U.S. culture and how to measure acculturation and investigate the long-term effects of length of stay in the United States on health outcomes in Haitian immigrants. There are high concentrations of Haitian immigrants in Miami, Florida; New York, New York; and Boston, Massachusetts (Sanon, Mohammed, \& McCullagh, 2014). Although immigrants enter the country with a lesser frequency of being overweight than the native population, the occurrence of obesity and diabetes rises with increased length of habitation in the United States (Afable-Munsuz et al., 2014; Oza-Frank et al., 2011; Venkatesh et al., 2013). Lu et al. (2015) identified that immigrant health outcomes can be influenced based on the individual's level of acculturation. Although I did not find a significant correlation between acculturation and BMI, more research is needed to investigate the relationship between acculturation, immigrant length of stay in the United States, and BMI.

This research adds knowledge to the literature by revealing that the Haitian population did not show declining health with acculturation and longer length of stay in the United States. Future researchers should examine additional predictors, such as level of education, dietary habits, and economic status as alternative explanations. Although I did not find a significant relationship between acculturation and length of stay in the United States and BMI, health care providers should use an acculturation scale to examine additional behavior measures on ethnic and cultural identity, cultural values (family cohesion), and cultural practices (English language proficiency; Rosas, Sanchez-Vaznaugh, \& Sanchez, 2015). The results of my study may affect positive social change by providing health care providers with a means of not only measuring acculturation in immigrant groups, but also by investigating the long-term effect of length of stay in the United States and the fluctuation in BMI change as a chronic disease prevention approach.

\section{Summary}

The aim of this study was to provide information to the Haitian immigrant population living in the United States about the risk of obesity and its ties to acculturation and length of stay in the host nation, as well as bringing greater insight to health care providers of the chronic diseases that may result from BMI change. The findings of this study are supported by similar studies but are not supported by other studies regarding the length of stay in the United States being related to BMI changes in Haitian immigrants. Because there were mixed results, more research is needed. In addition, the Schwartz model of acculturation was used as the conceptual framework to examine the relationship of acculturation through four mediators (ethnic and cultural identity, cultural values, cultural practices, and acculturation stress) and BMI change, which was measured by the bidimensional SMAS. This study reported new knowledge about the Haitian immigrants living in the Northeast metropolitan area and should generate longitudinal immigrant health research interest in order to establish Haitian immigrants' health status across length of stay in the United States. Immigrants' acculturation levels and length of stay in the United States have a long-term effect on BMI. Although there was not this significant correlation, I recommend that more research be conducted to investigate the correlation between acculturation, length of time in the United States, and BMI.

\section{References}

Afable-Munsuz, A., Mayed, E. R., Perez-Stable, I. J., \& Haan, M. N. (2014). Immigrant generation and diabetes risk among Mexican Americans: The Sacramento area Latino study on aging. American Journal of Public Health, 104, S243-S250. https://dx.doi.org/10.2105/AJPH.2012.300969 
Agbemenu, K. (2016). Acculturation and health behaviors of African immigrants living in the United States: An integrative review. Association of Black Nursing Faculty Journal, 27, 67-73.

Allen, A., Marcelin, L. H., Schmitz, S., Hausmann, V., \& Shultz, J. M. (2012). Earthquake impact on Miami Haitian Americans: The role of family/social connectedness. Journal of Loss \& Trauma, 17, 337-349. https://dx.doi.org/10.1080/15325024.2011.635577

Archuleta, A., J. (2015). Moderated linguistic acculturation and perceived group social capital on economic and occupational stress among people of Mexican descent. Journal of Ethnic and Cultural and Cultural Diversity in Social Work, 24, 130-147. https://doi.org/10.1080/15313204.2014.991978

Bates, D., Mächler, M., Bolker, B., \& Walker, S. (2014). Fitting linear mixed-effects models using lme4. arXiv preprint arXiv:1406.5823.

Buscemi, C, P. (2011). Acculturation: State of the science in nursing. Journal of Cultural Diversity, 18, 39-42.

Centers for Disease Control and Prevention (CDC). (2013). Adult obesity facts. Retrieved from www.cdc.gov/obesity/data/adult.html

Centers for Disease Control and Prevention (CDC). (2016). Overweight and obesity. Retrieved from www.cdc.gov/obesity/adult/

Centers for Disease Control and Prevention (CDC). (2018). Adult obesity facts. Retrieved from www.cdc.gov/obesity/data/adult.html

Cruz, Y., Hernandez-Lane, M., Cohello, J., \& Bautista, C. (2013). The effectiveness of a community health program in improving diabetes knowledge in the Hispanic population: Salud y Bienestar (Health and Wellness). Journal of Community Health, 38, 1124-1131. https://doi.org/10.1007/s10900-013-9722-9

Faul, F., Erdfelder, E., Buchner, A., \& Lang, A. G. (2014). G*power version 3.1.9. [Computer software]. Kiel, Germany: University of Kiel. Retrieved from http://www.gpower.hhu.de/en/html

Field, A. (2013). Discovering statistics using IBM SPSS Statistics (4th ed.). London, United Kingdom: Sage.

Flaskerud, J., \& Winslow, B. (2010). Vulnerable populations and ultimate responsibility. Issues in Mental Health Nursing, 31, 298-299. https://dx.doi.org/10.3109/01612840903308556

Gaskins, R. B., Baskin, M. L., \& Person, S. D. (2012). Language, duration of United States residency, and leisure time physical activity among women from the third National Health and Nutrition Examination Survey (NHANES III). Journal of Women's Health, 21, 1170-1179. https://dx.doi.org/10.1089/jwh.2012.3477

Hofmann, B. (2016). Obesity as a socially defined disease: Philosophical considerations and implications for policy and care. Health Care Analysis, 24, 86-100. https://dx.doi.org/10.1007/s10728-015-0291-1

Ialongo, C. (2016). Understanding the effect size and its measures. Biochemia Medica, 26, 150-163. https://dx.doi.org/10.11613/BM.2016.015

Kuo, C. H., B. (2014). Coping, acculturation, and psychological adaptation among migrants: A theoretical and empirical review and synthesis of the literature. Journal of Health Psychology and Behavioral Medicine, 2, 16-33 https://dx.doi.org/10.1080/21642850.2013.843459

Lê-Scherban, F., Albert, S. S., Osypuk, T. L., Sanchez, B. N., \& Diez Roux, A. V. (2014). Neighborhood ethnic composition, spatial assimilation, and change in body mass index over time among Hispanic and Chinese immigrants: Multi-ethnic study of atherosclerosis. American Journal of Public Health, 104, 2138-2146. https://dx.doi.org/10.2105/AJPH. 2014.302154 
Lind, L., Lind, P. M., Lejonklou, M. H., Dunder, L., Bergman, Å., Guerrero-Bosagna, C., ... Birnbaum, L. S. (2016). Uppsala consensus statement on environmental contaminants and the global obesity epidemic. Environmental Health Perspectives, 124, A81-A83. https://dx.doi.org/10.1289/ehp.1511115

Lu, S., Donglan, Z., Van Meijgaard, J., Macleod, K. E., \& Fielding, J. E. (2015). The interaction between an individual's acculturation and community factors on physical inactivity and obesity: A multilevel analysis. American Journal of Public Health, 105, 1460-1467.

https://dx.doi.org/10.2105/AJPH.2014.302541

Lubetkin, E. I., Zabor, E. C., Isaac, K., Brennessel, D., Kemeny, M. M., \& Hay, J. L. (2015). Health literacy, information seeking, and trust in information in Haitians. American Journal of Health Behavior, 39, 441-450. https://dx.doi.org/10.5993/AJHB.39.3.16

McDermott-Levy, R. (2009). Acculturation: A concept analysis for immigrant health. Holistic Nursing Practice, 23, 282-288. https://dx.doi.org/10.1097/HNP.oB013e3181b66cc7

Nguyen, H. H. D., Smith, C., Reynolds, G. L., \& Freshman, N. (2015). The effect of acculturation on obesity among foreign-born Asians residing in the United States. Journal of Immigrant Minor Health, 17, 389-399. https://dx.doi.org/10.1007/s10903-014-0027-6

Nyamathi, A., Koniak-Griffin, D., \& Greengold, B. (2007). Development of nursing theory and science in vulnerable populations research. Annual Review of Nursing Research, 25, 253-258. https://doi.org/10.1891/o739-6686.25.1.3

Oza-Frank, R., Stephenson, R., \& Venkat Narayan, K. (2011). Diabetes prevalence by length of residence among us immigrants. Journal of Immigrant \& Minority Health, 13, 1-8. https://dx.doi.org/10.10007/s10903-009-9283-2

Pituch, K. A., \& Stevens, J. P. (2015). Applied multivariate statistics for the social sciences: Analyses with SAS and IBM's SPSS. London, United Kingdom: Routledge.

Quick, J., \& Hall, S. (2015). Part three: The quantitative approach. Journal of Perioperative Practice, 25, 192196. https://doi.org/10.1177/175045891502501002

Ro, A., \& Bostean, G. (2015). Duration of U.S. stay and body mass among Latino and Asian immigrants: A test of theoretical pathways. Social Science \& Medicine, 144, 39-47. https://dx.doi.org/10.1016/j.socscimed.2015.09.001

Rosas, L. G., Sanchez-Vaznaugh, E., \& Sanchez, B., N. (2015). Nativity, U.S. length of residence, and BMI among diverse Asian American ethnic groups. Journal of Immigrant and Minority Health, 17, 14961503. https://dx.doi.org/10.1007/s10903-104-0096-6

Sanon, M., Mohammed, S. A., \& McCullagh, M. C. (2014). Definition and management of hypertension among Haitian immigrants: A qualitative study. Journal of Health Care for the Poor and Underserved, 25, 1067-1078. https://doi.org/10.1353/hpu.2014.0147

Schwartz, S. J. Kim, S. Y., Whitebourne, S. K., Zamboanga, B. L., Weisskirch, R. S. Forthun, L., \& Luyckx, K. (2013). Converging identities: Dimensions of acculturation and personal identity status among immigrant college students. Cultural Diversity \& Ethnic Minority Psychology, 19, 155-165. https://dx.doi.org/ 10.1037/ao030753

Schulz, J., \& Batalova, J. (2017, August 2). Haitian immigrants in the United States. The Online Journal of Migration Policy Institute. Retrieved from http://migrationpolicy.org/article/haitian-immigrantsunited-states 
Stephenson, M (2000). Development of validation of the Stephenson Multigroup Acculturation Scale (SMAS). Psychology Assessment, 12, 77-88. https://dx.doi.org/10.1037/10403590.12.1.77

Tsujimoto, T., Kajio, H., \& Sugiyama. T. (2016). Obesity, diabetes, and length of time in the United States: Analysis of National Health and Nutrition Examination Survey 1999 to 2012. Journal of Medicine, 95, 35. https://dx.doi.org/10.1097/MD.0000000000004578

Venkatesh, S., Weatherspoon, L., Kaplowitz, S., \& Song, W. (2013). Acculturation and glycemic control of Asian Indian adults with Type 2 diabetes. Journal of Community Health, 38, 78-85. https://dx.doi.org/10.1007/s10900-012-9584-6

Weisskirch, R. (2013). Family relationships, self-esteem, and self-efficacy among language brockering Mexican American emerging adults. Journal of Child \& Family Studies, 22, 1147-1144. https://dx.doi.org/10.1007/s10826-012-9678-x

Xi, J., Takyi, B., \& Lamptey, E. (2015). Are recent immigrants larger than earlier ones at their arrival? Cohort variation in initial BMI among U.S. immigrants, 1989-2011. Journal of Immigrant and Minority Health, 17, 1854-1862. https://dx.doi.org/10.1007/s10903-014-0129-1

Zhang, Y. L., \& Tsai, J. L. (2014). The assessment of acculturation, enculturation, and culture in AsianAmerican samples. In L. Benuto, N. Thaler, \& B. Leany (Eds.), Guide to psychological assessment with Asians (pp. 75-101). New York, NY: Springer. https://dx.doi.org/10.1007/978-1-4939-0796o_6 


\section{Contact Hour Information}

\section{Goal Statement}

The goal of this nursing continuing professional development activity is to provide nurses with information about the impact of acculturation on the body mass index of Haitian American immigrants.

\section{Outcome Measure}

After completing this nursing continuing professional development activity, nurses will gain knowledge of the impact of acculturation on body mass index in Haitian American immigrant populations.

\section{Disclosure}

The author and planners of this nursing continuing professional development activity have disclosed no relevant relationships with commercial interests in pertaining to any information within this article.

Contact Hours: 0.6

Contact Hour Expiration Date: September 27, 2021

\section{How to Obtain Contact Hours for this Activity}

1. Click on the following URL (link) or copy and paste it to Google Chrome or Firefox browser: https://waldenuniversitycne.rievent.com/a/BXKMQV

2. Complete the registration process and respond to the post-test questions (passing score is $80 \%$ ).

3. Complete the evaluation.

4. Print or download and save your certificate.

Walden University is accredited as a provider of nursing continuing professional development by the American Nurses Credentialing Center's Commission on Accreditation. Po469.

\section{O | JENHP}

The Journal of Excellence in Nursing and Healthcare

Practice welcomes manuscripts focusing on topics such as national and international workforce issues, quality improvement projects, evidence-based practice initiatives, nursing research studies, interprofessional practice, educational issues, telehealth, improvements in technology, and the impact of social change in society. 